\title{
Generalized Neutrosophic Set and Generalized Neutrosophic Topological Spaces
}

\author{
A. A. Salama ${ }^{1, *}$, S. A. Alblowi ${ }^{2}$ \\ ${ }^{1}$ Department of Mathematics and Computer Science, Faculty of Sciences, Port Said University, Egypt \\ ${ }^{2}$ Department of Mathematics, King Abdulaziz University,Jeddah Saudi Arabia
}

\begin{abstract}
In this paper we introduce definitions of generalized neutrosophic sets. After given the fundamental defin itions of generalized neutrosophic set operations, we obtain several properties, and discussed the relationship between generalized neutrosophic sets and others. Finally, we extend the concepts of neutrosophic topological space [9], intuition istic fuzzy topological space [5, 6], and fu zzy topological space [4] to the case of generalized neutrosophic sets. Possible application to GIS topology rules are touched upon.
\end{abstract}

Keywo rds Neutrosophic Set, Generalized Neutrosophic Set, Neutrosophic Topology

\section{Introduction}

Neutrosophy has laid the foundation for a whole family of new mathematical theories generalizing both their classical and fuzzy counterparts, such as a neutrosophic set theory. The fuzzy set was introduced by Zadeh [10] in 1965, where each ele ment had a degree of membership. The intuitionstic fuzzy set (Ifs for short) on a universe X was introduced by K. Atanassov [1, 2, 3] in 1983 as a generalization of fuzzy set, where besides the degree of me mbership and the degree of non-membership of each element. After the introduction of the neutrosophic set concept $[7,8,9]$. In this paper we introduce definitions of generalized neutrosophic sets. After given the fundamental defin itions of generalized neutrosophic set operations, we obtain several properties, and discussed the relationship between generalized neutrosophic sets and others. Finally, we extend the concepts of neutrosophic topological space [9].

\section{Terminologies}

We recollect some relevant basic preliminaries, and in particular, the work of Smarandache in [7, 8], Atanassov in [1, 2, 3] and Salama [9]. Smarandache introduced the neutrosophic components $\mathrm{T}$, I, F which represent the membership, indeterminacy, and non-membership values respectively, where $] 0^{-}, 1^{+}[$is nonstandard unit interval.

Definition. $[7,8]$

* Corresponding author:

drsalama44@gmail.com (A. A.Salama)

Published online at http://journal.sapub.org/computer

Copyright (C) 2012 Scientific \& Academic Publishing. All Rights Reserved
Let $\mathrm{T}, \mathrm{I}, \mathrm{F}$ be real standard or nonstandard subsets of ] $0^{-}, 1^{+}[$, with

Sup_T=t_sup, inf_T $=$ t_inf

Sup_I $=$ i_sup, inf_ $\mathrm{I}=\mathrm{i} \_$inf

Sup_F=f_sup, inf_F $=\mathrm{f}_{-}$inf

$\mathrm{n}$-sup $=\mathrm{t} \_$sup $+\mathrm{i}_{-}$sup $+\mathrm{f} \_$sup

$\mathrm{n}$-inf $=\mathrm{t}$ inf $+\mathrm{i}$ inf $+\mathrm{f}$ - inf,

T, I, F are called neutrosophic components

Definition [9]

Let $\mathrm{X}$ be a non-empty fixed set. $A$ neutrosophic set (NS for short) $A$ is an object having the form $A=\left\{\left\langle x, \mu_{A}(x), \sigma_{A}(x), \gamma_{A}(x)\right\rangle: x \in X\right\} \quad$ Where $\mu_{A}(x), \sigma_{A}(x)$ and $\gamma_{A}(x)$ which represent the degree of member ship function (namely $\mu_{A}(x)$ ), the degree of indeterminacy (namely $\sigma_{A}(x)$ ), and the degree of non-member ship (namely $\gamma_{A}(x)$ ) respectively of each element $x \in X$ to the set $A$.

Definition [9].

The NSS $0_{N}$ and $1_{N}$ in $X$ as follows:

$0_{N}$ may be defined as:
$\left(0_{1}\right) \quad 0_{N}=\{\langle x, 0,0,1\rangle: x \in X\}$
$\left(0_{2}\right) \quad 0_{N}=\{\langle x, 0,1,1\rangle: x \in X\}$
$\left(0_{3}\right) \quad 0_{N}=\{\langle x, 0,1,0\rangle: x \in X\}$
$\left(0_{4}\right) \quad 0_{N}=\{\langle x, 0,0,0\rangle: x \in X\}$
$1_{N}$ may be defined as:
$\left(1_{1}\right) 1_{N}=\{\langle x, 1,0,0\rangle: x \in X\}$ 

$\left(1_{2}\right) 1_{N}=\{\langle x, 1,0,1\rangle: x \in X\}$
$\left(1_{3}\right) 1_{N}=\{\langle x, 1,1,0\rangle: x \in X\}$
$\left(1_{4}\right) 1_{N}=\{\langle x, 1,1,1\rangle: x \in X\}$

\section{Generalized Neutrosophic Sets}

We shall now consider some possible definitions for basic concepts of the generalized neutrosophic set.

\section{Definition}

Let $\mathrm{X}$ be a non-empty fixed set. $A$ generalized neutrosophic set (GNS for short) $A$ is an object having the form $A=\left\{\left\langle x, \mu_{A}(x), \sigma_{A}(x), \gamma_{A}(x)\right\rangle: x \in X\right\}$ Where $\mu_{A}(x), \sigma_{A}(x)$ and $\gamma_{A}(x)$ which represent the degree of member ship function (namely $\mu_{A}(x)$ ), the degree of indeterminacy (namely $\sigma_{A}(x)$ ), and the degree of non-member ship (namely $\gamma_{A}(x)$ ) respectively of each element $x \in X$ to the set $A$ where the functions satisfy the condition $\mu_{A}(x) \wedge \sigma_{A}(x) \wedge v_{A}(x) \leq 0.5$.

\section{Remark}

A generalized neutros ophic

$A=\left\{<x, \mu_{A}(x), \sigma_{A}(x), \gamma_{A}(x)>: x \in X\right\}$ can be identified to an ordered triple $\left\langle\mu_{A}, \sigma_{A}, \gamma_{A}>\text { in }\right]^{-} 0,1^{+}[$on.X, where the trip le functions satisfy the condition $\mu_{A}(x) \wedge \sigma_{A}(x) \wedge \nu_{A}(x) \leq 0.5$

\section{Remark}

For the sake of simp licity, we shall use the symbol $A=<x, \mu_{A}, \sigma_{A}, \gamma_{A}>$ for the G $N S$ $A=\left\{<x, \mu_{A}(x), \sigma_{A}(x), \gamma_{A}(x)>: x \in X\right\}$

\section{Example}

Every GIFS $A$ a non-empty set $X$ is obviously on GNS having the form

$$
A=\left\{<x, \mu_{A}(x), 1-\left(\mu_{A}(x)+\gamma_{A}(x)\right), \gamma_{A}(x)>: x \in X\right\}
$$

\section{Definiti on}

Let $A=\left\langle\mu_{A}, \sigma_{A}, \gamma_{A}\right\rangle$ a GNSS on $X$, then the complement of the set $A(C(A)$, for short ) maybe defined as three kinds of complements

$\left(C_{1}\right) C(A)=\left\{\left\langle x, 1-\mu_{A}(x), \sigma_{A}(x), 1-v_{A}(x)\right\rangle: x \in X\right\}$,

$\left(C_{2}\right) \quad C(A)=\left\{\left\langle x, \gamma_{A}, \sigma_{A}(x), \mu_{A}(x)\right\rangle: x \in X\right\}$

$\left(C_{3}\right) \quad C(A)=\left\{\left\langle x, \gamma_{A}, 1-\sigma_{A}(x), \mu_{A}(x)\right\rangle: x \in X\right\}$

One can define several relations and operations between GNSS as follows:

\section{Definiti on}

Let $X$ be a non-empty set, and GNSS $A$ and $B$ in the form $A=\left\langle x, \mu_{A}(x), \sigma_{A}(x), \gamma_{A}(x)\right\rangle$,

$B=\left\langle x, \mu_{B}(x), \sigma_{B}(x), \gamma_{B}(x)\right\rangle$, then we may consider two possible definitions for subsets $(A \subseteq B)$

$(A \subseteq B)$ may be defined as

$A \subseteq B \Leftrightarrow \mu_{A}(x) \leq \mu_{B}(x), \gamma_{A}(x) \geq \gamma$ and $\sigma_{A}(x) \leq \sigma_{B}(x)$

$\forall x \in X$

$A \subseteq B \Leftrightarrow \mu_{A}(x) \leq \mu_{B}(x), \gamma_{A}(x) \geq \gamma_{B}(x)$ and $\sigma_{A}(x) \geq \sigma_{B}(x)$

\section{Proposition}

For any generalized neutrosophic set $A$ the following are holds

$$
0_{N} \subseteq A, \quad 0_{N} \subseteq 0_{N}
$$$$
A \subseteq 1_{N}, \quad 1_{N} \subseteq 1_{N}
$$

\section{Definition}

Let $X$ be a non-empty set, and

$A=<x, \mu_{A}(x), \gamma_{A}(x), \sigma_{A}(x)>$,

$B=<x, \mu_{B}(x), \sigma_{B}(x), \gamma_{B}(x)>$ are GNSS Then

$A \cap B$ maybe defined as:

(I $\left.I_{1}\right) A \cap B=<x, \mu_{A}(x) \cdot \mu_{B}(x), \sigma_{A}(x) \cdot \sigma_{B}(x)$,

$\gamma_{A}(x) \cdot \gamma_{B}(x)>$

(I $\left.I_{2}\right) A \cap B=<x, \mu_{A}(x) \wedge \mu_{B}(x), \sigma_{A}(x) \wedge \sigma_{B}(x)$,

$\gamma_{A}(x) \vee \gamma_{B}(x)>$

$\left(I_{3}\right) A \cap B=<x, \mu_{A}(x) \wedge \mu_{B}(x), \sigma_{A}(x) \vee \sigma_{B}(x)$,

$\gamma_{A}(x) \vee \gamma_{B}(x)>$

$A \cup B$ may be defined as:

$\left(U_{1}\right) \quad A \cup B=<x, \mu_{A}(x) \vee \mu_{B}(x), \sigma_{A}(x) \vee \sigma_{B}(x)$,

$\gamma_{A}(x) \wedge \gamma_{B}(x)>$

$\left(U_{2}\right) \quad A \cup B=<x, \mu_{A}(x) \vee \mu_{B}(x), \sigma_{A}(x) \wedge \sigma_{B}(x)$,

$\gamma_{A}(x) \wedge \gamma_{B}(x)>$

[]$A=<x, \mu_{A}(x), \sigma_{A}(x), 1-\mu_{A}(x)>$

$<>A=<x, 1-\gamma_{A}(x), \sigma_{A}(x), \gamma_{A}(x)>$

Example.3.2. Let $X=\{a, b, c, d, e\}$ and $A=\left\langle x, \mu_{A}, \sigma_{A}, v_{A}\right\rangle$ given by:

\begin{tabular}{|c|c|c|c|c|}
\hline $\mathrm{X}$ & $\mu_{A}(x)$ & $v_{A}(x)$ & $\sigma_{A}(x)$ & $\mu_{A}(x) \wedge v_{A}(x) \wedge \sigma_{A}(x)$ \\
\hline $\mathrm{a}$ & 0.6 & 0.3 & 05 & 0.3 \\
\hline $\mathrm{b}$ & 0.5 & 0.3 & 0.6 & 0.3 \\
\hline $\mathrm{c}$ & 0.4 & 0.4 & 0.5 & 0.4 \\
\hline $\mathrm{d}$ & 0.3 & 0.5 & 0.3 & 0.3 \\
\hline $\mathrm{e}$ & 0.3 & 0.6 & 0.4 & 0.3 \\
\hline
\end{tabular}

Then the family $G=\left\{O_{\sim}, A\right\}$ is an GNSS on X.

We can easily generalize the operations of generalized intersection and union in definition 3.4 to arbitrary family of GNSS as follow:

\section{Definition}

Let $\{A j: j \in J\}$ be a arbitrary family of NSS in $X$, then

$\cap A j$ maybe defined as:

1) $\cap A j=\left\langle x, \underset{j \in J}{\wedge} \mu_{A j}(x), \underset{j \in J}{\wedge} \sigma_{A j}(x), \vee \gamma_{A j}(x)\right\rangle$ 
2) $\cap A j=\left\langle x, \wedge \mu_{A j}(x), \vee \sigma_{A j}(x), \vee \gamma_{A j}(x)\right\rangle$

$\cup A j$ maybe defined as:

1) $\cup A_{j}=\left\langle x \underset{j \in J}{\vee} \mu_{A_{j}}, \wedge \sigma_{A_{j}}, \wedge v_{A_{j}}\right\rangle$

2) $\cup A_{j}=\left\langle x, \underset{j \in J}{\vee} \mu_{A_{j}}, \vee \sigma_{A_{j}}, \wedge v_{A_{j}}\right\rangle$

\section{Definition}

Let $A$ and $B$ are generalized neutrosophic sets then $A \mid B$ may be defined as

$A \mid B=\left\langle x, \mu_{A} \wedge \gamma_{B}, \sigma_{A}(x) \sigma_{B}(x), \gamma_{A} \vee \mu_{B}(x)\right\rangle$

\section{Proposition}

For all $A, B$ two generalized neutrosophic sets then the following are true

i) $C(A \cap B)=C(A) \cup C(B)$

ii) $C(A \cup B)=C(A) \cap C(B)$

\section{Generalized Neutrosophic Topological Spaces}

Here we extend the concepts of and intuitionistic fuzzy topological space [5, 7], and neutrosophic topological Space [9] to the case of generalized neutrosophic sets.

\section{Definition}

A generalized neutrosophic topology (GNT for short) an a non empty set $X$ is a family $\tau$ of generalized neutrosophic subsets in $X$ satisfying the following axio ms

$\left(G N T_{1}\right) O_{N}, 1_{N} \in \tau$,

$\left(G N T_{2}\right) G_{1} \cap G_{2} \in \tau$ for any $G_{1}, G_{2} \in \tau$,

$\left(G N T_{3}\right) \cup G_{i} \in \tau \quad \forall\left\{G_{i}: i \in J\right\} \subseteq \tau$

In this case the pair $(X, \tau)$ is called a generalized neutrosophic topological space (GNTS for short) and any neutrosophic set in $\tau$ is known as neuterosophic open set (NOS for short) in $X$. The elements of $\tau$ are called open generalized neutrosophic sets, A generalized neutrosophic set $\mathrm{F}$ is closed if and only if it $\mathrm{C}(\mathrm{F})$ is generalized neutrosophic open.

Remark A generalized neutrosophic topological spaces are very natural generalizations of intuitionistic fuzzy topological spaces allow more general functions to be members of intuitionistic fuzzy topology.

\section{Example}

$$
\begin{aligned}
& \text { Let } X=\{x\} \text { and } \\
& A=\{\langle x, 0.5,0.5,0.4\rangle: x \in X\} \\
& B=\{\langle x, 0.4,0.6,0.8\rangle: x \in X\} \\
& D=\{\langle x, 0.5,0.6,0.4\rangle: x \in X\} \\
& C=\{\langle x, 0.4,0.5,0.8\rangle: x \in X\}
\end{aligned}
$$

Then the family $\tau=\left\{O_{n}, 1_{n}, A, B, C, D\right\}$ of G NSs in $X$ is generalized neutrosophic topology on $X$

\section{Example}

Let $\left(X, \tau_{0}\right)$ be a fuzzy topological space in Changes [4] sense such that $\tau_{0}$ is not indiscrete suppose now that $\tau_{0}=\left\{0_{N}, 1_{N}\right\} \cup\left\{V_{j}: j \in J\right\}$ then we can construct two G NTSS on $X$ as follows

$$
\begin{gathered}
\tau_{0}=\left\{0_{N}, 1_{N}\right\} \cup\left\{<x, V_{j}, \sigma(x), 0>: j \in J\right\} \\
\tau_{0}=\left\{0_{N}, 1_{N}\right\} \cup\left\{<x, V_{j}, 0, \sigma(x), 1-V_{j}>: j \in J\right\}
\end{gathered}
$$

\section{Proposition}

Let $(X, \tau)$ be a GNT on $X$, then we can also construct several GNTSS on $X$ in the following way:

a) $\tau_{o, 1}=\{[] G: G \in \tau\}$,

b) $\tau_{o, 2}=\{<>G: G \in \tau\}$,

\section{Proof a)}

$\left(G N T_{1}\right)$ and $\left(G N T_{2}\right)$ are easy.

$\left(G N T_{3}\right)$ Let $\left\{[] G_{j}: j \in J, G_{j} \in \tau\right\} \subseteq \tau_{0,1}$. Since

$\cup G_{j}=\left\{\left(x, \vee \mu_{G_{j}}, \vee \sigma_{G_{j}}, \wedge \gamma_{G_{j}}\right)\right\} \operatorname{or}\left\{\left(x, \vee \mu_{G_{j}}, \wedge \sigma_{G_{j}}, \wedge \gamma_{G_{j}}\right)\right\} \operatorname{or}\left\{\left(x, \vee \mu_{G_{j}}, \wedge \sigma_{G_{j}}, \vee \gamma_{G_{j}}\right)\right\} \in \tau$,

we have

$\cup\left([] G_{j}\right)=\left\{x, \vee \mu_{G_{j}}, \vee \sigma_{G_{j}}, \wedge\left(1-\mu_{G_{j}}\right)\right\} o r\left\{x, \vee \mu_{G_{j}}, \vee \sigma_{G_{j}},\left(1-\vee \mu_{G_{j}}\right)\right\} \in \tau_{0,1}$

This similar to (a)

\section{Definition}

Let $\left(X, \tau_{1}\right),\left(X, \tau_{2}\right)$ be two generalized neutrosophic topological spaces on $X$. Then $\tau_{1}$ is said be contained in $\tau_{2}$ (in symbols $\tau_{1} \subseteq \tau_{2}$ ) if $G \in \tau_{2}$ for each $G \in \tau_{1}$. In this case, we also say that $\tau_{1}$ is coarser than $\tau_{2}$.

\section{Proposition}

Let $\left\{\tau_{j}: j \in J\right\}$ be a family of NTSS on $X$. Then $\cap \tau_{j}$ is A generalized neutrosophic topology on $X$.Furthermore, $\cap \tau_{j}$ is the coarsest NT on $X$ containing all. $\tau_{j}, \mathrm{~s}$

Proof. Obvious

\section{Definition}

The complement of $\boldsymbol{A}$ (C (A) for short) of NOS. $\boldsymbol{A}$ is called a generalized neutrosophic closed set (G NCS for short) in $X$.

Now, we define generalized neutrosophic closure and interior operations in generalized neutrosophic topological spaces:

\section{Definition}

Let $(X, \tau)$ be G NTS and $A=<x, \mu_{A}(x), \gamma_{A}(x), \sigma_{A}(x)>$ be a $\mathrm{G} N S$ in $X$.

Then the generalized neutrosophic closer and generalized neutrosophic interior of Aare defined by

$\mathrm{G} \operatorname{NCl}(A)=\cap\{K: K$ is an $\mathrm{NCS}$ in $\mathrm{X}$ and $\mathrm{A} \subseteq \mathrm{K}\}$

$\mathrm{G}_{\operatorname{NInt}}(A)=\cup\{G: G$ is an $\mathrm{NOS}$ in $\mathrm{X}$ and $\mathrm{G} \subseteq \mathrm{A}\}$.It can be also shown that

It can be also shown that $N C l(A)$ is $N C S$ and $\operatorname{NInt}(A)$ is a $\mathrm{G} N O S$ in $X$

$A$ is in $X$ if and only if $\mathrm{G}_{\operatorname{NCl}(A)}$.

$A$ is $\mathrm{G} \mathbf{N} \quad$ in $X$ if and only if $\mathrm{G} \operatorname{NInt}(A)=A$.

\section{Proposition}


For any generalized neutrosophic set $A$ in $(x, \tau)$ we have

(a) $\mathrm{G} \operatorname{NCl}(C(A)=C(G N \operatorname{Int}(A)$,

(b) $\mathrm{G} \operatorname{NInt}(C(A))=C(G N C l(A))$.

Proof.

Let $\left.A=\left\{<x, \mu_{A}, \sigma_{A}, v_{A}\right\rangle: x \in X\right\}$ and suppose that the family of generalized neutrosophic subsets contained in $A$ are indexed by the family if G NSS contained in $A$ are indexed by the family $A=\left\{<x, \mu_{G_{i}}, \sigma_{G_{i}}, v_{G_{i}}>: i \in J\right\}$. Then we see that $\operatorname{GNInt}(A)=\left\{<x, \vee \mu_{G_{i}}, \vee \sigma_{G_{i}}, \wedge v_{G_{i}}>\right\}$ and hence $C(G N \operatorname{Int}(A))=\left\{<x, \wedge \mu_{G_{i}}, \vee \sigma_{G_{i}}, \vee v_{G_{i}}>\right\}$. Since $C(A)$ and $\mu_{G_{i}} \leq \mu_{A}$ and $v_{G_{i}} \geq v_{A}$ for each $i \in J$, we obtaining $C(A)$. i.e

$\operatorname{GNCl}(C(A))=\left\{<x, \wedge v_{G_{i}}, \vee \sigma_{G_{i}}, \vee \mu_{G_{i}}>\right\}$. Hence $\operatorname{GNCl}(C(A)=C(G N \operatorname{Nnt}(A)$, follows immediately

This is analogous to (a).

\section{Proposition}

Let $(x, \tau)$ be a GNS and $A, B$ be two neutrosophic sets in $X$. Then the following properties hold:

$\operatorname{GNInt}(A) \subseteq A$,

$A \subseteq \operatorname{GNCl}(A)$,

$A \subseteq B \Rightarrow G N \operatorname{Int}(A) \subseteq G N \operatorname{Int}(B)$,

$A \subseteq B \Rightarrow G N C l(A) \subseteq G N C l(B)$,

$\operatorname{GNInt}(G N \operatorname{Int}(A))=G N \operatorname{Int}(A) \wedge G \operatorname{GInt}(B)$,

$\operatorname{GNCl}(A \cup B)=G N C l(A) \vee G N C l(B)$,

$\operatorname{GNInt}\left(1_{N}\right)=1_{N}$,

$\operatorname{GNCl}\left(O_{N}\right)=O_{N}$,

Proof (a), (b) and (e) are obvious (c) follows from (a) and
Definitions.

\section{REFERENCES}

[1] K. Atanassov, intuitionistic fuzzy sets, in V.Sgurev, ed., Vii ITKRS Session, Sofia(June 1983 central Sci. and Techn. Library, Bulg. Academy of Sciences( 1984)).

[2] K. Atanassov, intuitionistic fuzzy sets, Fuzzy Sets and Systems 20(1986)87-96.

[3] K. Atanassov, Review and new result on intuitionistic fuzzy sets , preprint IM-MFAIS-1-88, Sofia, 1988.

[4] C.L. Chang, Fuzzy Topological Spaces, J. Math. Anal. Appl. 24 (1968)182-1 90.

[5] Dogan Coker, An introduction to intuitionistic fuzzy topological spaces, Fuzzy Sets and Systems. 88(1997)81-89.

[6] Reza Saadati, Jin HanPark, On the intuitionistic fuzzy topological space, Chaos, Solitons and Fractals 27(2006)331-344 .

[7] Florentin Smarandache, Neutrosophy and Neutrosophic Logic, First International Conference on Neutrosophy , Neutrosophic Logic, Set, Probability, and Statistics University of New Mexico, Gallup, NM 87301, USA(2002), smarand@unm.edu

[8] F. Smarandache. A Unify ing Field in Logics: Neutrosophic Logic. Neutrosophy, Neutrosophic Set, Neutrosophic Probability. American Research Press, Rehoboth, NM, 1999.

[9] A.A. Salama and S.A. AL-Blowi, NEUTROSOPHIC SET and NEUTROSOPHIC TOPOLOGICAL SPACES, IOSR Journal of Math. ISSN:2278-5728.Vol.(3) ISSUE4PP31-35(2012)

[10] L.A. Zadeh, Fuzzy Sets, Inform and Control 8(1965)338-353. 